\title{
On the Exact WKB Analysis of Second Order Linear Ordinary Differential Equations with Simple Poles
}

By

\author{
Tatsuya KoIKE*
}

\section{§1. Introduction}

The principal aim of this paper is to do an exact WKB-theoretic study of a linear differential equation of the form

$$
\left(-\frac{d^{2}}{d x^{2}}+\eta^{2} Q(x)\right) \psi=0
$$

on a neighborhood of a simple pole of the potential $Q(x)$. Here and in what follows $\eta$ denotes a large parameter. Such a study of (1.1) has been completely done near a point where $Q(x)$ is holomorphic and has a simple zero (i.e., near a simple turning point) or near a point where $Q(x)$ has a double pole. (See [9], [1], [2], and references cited there.) However no exact WKB-theoretic study of (1.1) near a simple pole has ever been done. (See [4] and [8] for some nonexact WKB-theoretic study.) The difficulty arises from the fact that a simple pole plays also a role of turning points in the exact $\mathrm{WKB}$ analysis as it appears as a consequence of the confluence of a simple turning point and a double pole. (Cf. $\S 3$ below.) In this paper we establish a connection formula for the Borel transform of WKB solutions of (1.1) near a simple pole of $Q(x)$, using the canonical equation found in [5].

The plan of this paper is as follows: In $\S 2$, after introducing some basic notions and notations in the exact WKB analysis, we state our main theorem on the connection formula for the Borel transform of WKB solutions of (1.1) near a simple pole. In $\S 3$ we first recall the results in [5] which enable us to bring the equation (1.1) to the canonical form

Communicated by T. Kawai December 10, 1999.

1991 Mathematics Subject Classification(s): 34M60

* Research Institute for Mathematical Sciences, Kyoto University, Kyoto 606-8502, Japan. 


$$
\left(-\frac{d^{2}}{d x^{2}}+\eta^{2}\left(\frac{1}{x}+\eta^{-2} \frac{\lambda}{x^{2}}\right)\right) \psi=0
$$

near a simple pole of $Q(x)$ through a "formal coordinate transformation". As a matter of fact, having the application of our results to the study of (1.1) with a periodic potential $Q$ in mind, we discuss somewhat more general equations of the form

$$
\left(-\frac{d^{2}}{d x^{2}}+\eta^{2}\left(\frac{Q_{0}(x)}{x}+\eta^{-2} \frac{Q_{2}(x)}{x^{2}}\right)\right) \psi=0,
$$

where $Q_{0}(x)$ and $Q_{2}(x)$ denote holomorphic functions near the origin satisfying $Q_{0}(0) \neq 0$. Note that, if the potential has the form $P(\cos x)$ with a polynomial $P(z)$, then a coordinate transformation $z=\cos x$ will bring (1.1) to the form

$$
\left(-\frac{d^{2}}{d z^{2}}+\eta^{2}\left(\frac{P(z)}{1-z^{2}}-\eta^{-2} \frac{z^{2}+2}{4\left(1-z^{2}\right)^{2}}\right)\right) \psi=0 .
$$

Although the construction of a "formal coordinate transformation" has been done in parallel with [1] where a simple turning point is discussed, giving an analytic meaning to the "formal transformation" is much more difficult in our case; as the singularity spectrum of the Borel transform of a WKB solution of (1.2) is so wide that we cannot employ the microlocal approach used in [1]. We overcome this difficulty by making full use of the explicit form of WKB solutions of (1.2). Having the explicit form of the Borel transform of WKB solutions of (1.2) in mind, we study the Borel transform of the "formally transformed" WKB solutions of (1.2) that give WKB solutions of (1.3) and establish the connection formula. As it will become evident in the course of our discussion, the resulting connection formula is a variant of Gauss' connection formula for hypergeometric functions (with some particular parameters, cf. §3). In the final section we give a proof of key estimates.

Some applications of the results in this paper to Heun's equation can be found in [6], and applications to the case where $Q(x)$ is periodic will be given in our subsequent article.

I wish to express my sincere gratitude to Professor Takahiro Kawai and Professor Yoshitsugu Takei for helpful suggestions and stimulating discussions. I would also like to thank Professor Takashi Aoki for valuable comments.

\section{§2. Statement of the Main Results}

In what follows we discuss the equation (1.3):

$$
\left(-\frac{d^{2}}{d x^{2}}+\eta^{2}\left(\frac{Q_{0}(x)}{x}+\eta^{-2} \frac{Q_{2}(x)}{x^{2}}\right)\right) \psi=0 .
$$

To state our main results we prepare some notations. (We follow [2].) 
A WKB solution of (2.1) is, by definition, a solution of (2.1) of the following form:

$$
\psi_{ \pm}(x, \eta)=\exp \left(\int^{x} S_{ \pm}(x, \eta) d x\right)
$$

where

$$
S_{ \pm}(x, \eta)= \pm \eta S_{-1}(x)+S_{0}(x) \pm \eta^{-1} S_{1}(x)+\cdots
$$

satisfies the Riccati equation

$$
S^{2}+\frac{\partial S}{\partial x}=\eta^{2}\left(\frac{Q_{0}(x)}{x}+\eta^{-2} \frac{Q_{2}(x)}{x^{2}}\right)
$$

Note that

$$
S_{-1}(x)=\sqrt{\frac{Q_{0}(x)}{x}}
$$

and that $S_{2 j-1}(x)(j=0,1,2, \ldots)$ has the singularity of square-root type at the origin. Note also that, denoting

$$
S_{\text {odd }}=\sum_{j \geq 0} S_{2 j-1} \eta^{1-2 j} \quad \text { and } \quad S_{\text {even }}=\sum_{j \geq 0} S_{2 j} \eta^{-2 j}
$$

we have

$$
S_{\text {even }}=-\frac{1}{2} \frac{\partial}{\partial x} \log S_{\text {odd }}
$$

Thus we can introduce a normalized WKB solution of the following form:

$$
\psi_{ \pm}=\frac{1}{\sqrt{S_{\text {odd }}}} \exp \left( \pm \int_{0}^{x} S_{\text {odd }} d x\right) .
$$

Here the integral in the right-hand side of (2.8) should be regarded as a contour integral. In the following we say the WKB solutions (2.8) as those normalized at the origin.

WKB solutions (2.8) are not convergent in general. To overcome this problem we employ the exact WKB analysis initiated by Voros ([9], see also [3]), which is based on the Borel summation method.

To consider the Borel sum of WKB solutions we first consider their Borel transform; for an infinite series of the form

$$
\phi(x, \eta)=e^{s(x) \eta} \sum_{j=0}^{\infty} \phi_{j}(x) \eta^{-j-\alpha} \quad(\alpha \in \mathbf{C} \backslash \mathbf{Z}),
$$


where $s(x)$ and $\phi_{j}(x)(j \geq 0)$ are holomorphic functions in a common domain $U$ of $\mathbb{C}$, its Borel transform $\phi_{B}(x, y)$ is, by definition,

$$
\phi_{B}(x, y)=\sum_{j=0}^{\infty} \frac{\phi_{j}(x)}{\Gamma(j+\alpha)}(y+s(x))^{j+\alpha-1}
$$

Note that WKB solutions (2.8) can be expanded in the form of (2.9); for example, in the case of $\psi_{+}$we take

$$
s(x)=\int_{0}^{x} \sqrt{\frac{Q_{0}(x)}{x}} d x
$$

and $\alpha=1 / 2$. If for any compact set $K$ in $U$ there exist constants $A_{K}$ and $C_{K}$ for which

$$
\sup _{K}\left|\phi_{j}(x)\right| \leq A_{K} C_{K}^{j} j ! \quad(j=0,1,2, \ldots)
$$

holds (and WKB solutions do satisfy this condition), then $\phi_{B}(x, y)$ defines an analytic function on a neighborhood of $(x,-s(x)) \in \mathbb{C}^{2}$. If $\phi_{B}(x, y)$ can, further, be analytically continued to a neighborhood of

$$
\{(x, y) \in U \times \mathbb{C} ; \Im y=\Im(-s(x)), \mathfrak{R} y \geq \mathfrak{R}(-s(x))\}
$$

and the integral

$$
\int_{-s(x)}^{\infty} e^{-\eta y} \phi_{B}(x, y) d y
$$

converges where the path of integration is parallel to the positive real axis, we say $\phi(x, \eta)$ is Borel summable and call the integral (2.14) the Borel sum of (2.9).

Using these terminologies, we now state the main theorem:

Theorem 2.1. There exists a positive constant $r_{0}$ for which the following hold:

(i) $(y+s(x))^{1 / 2} \psi_{+, B}(x, y) \quad$ (resp. $\left.(y-s(x))^{1 / 2} \psi_{-, B}(x, y)\right)$ is convergent and defines a holomorphic function in

$$
\begin{gathered}
W_{+}\left(r_{0}\right)=\left\{(x, y) \in \mathbb{C}^{2} ; 0<|x|<r_{0},|y+s(x)|<2|s(x)|\right\} \\
\left(\text { resp. } W_{-}\left(r_{0}\right)=\left\{(x, y) \in \mathbb{C}^{2} ; 0<|x|<r_{0},|y-s(x)|<2|s(x)|\right\}\right) .
\end{gathered}
$$

(ii) $\psi_{+, B}(x, y)$ (resp. $\psi_{-, B}(x, y)$ ) can be analytically continued and defines a multi-valued analytic function in $W_{-}\left(r_{0}\right) \backslash\{y=s(x)\} \quad$ (resp. $\left.W_{+}\left(r_{0}\right) \backslash\{y=-s(x)\}\right)$.

(iii) The discontinuity of $\psi_{+, B}(x, y)$ (resp. $\psi_{-, B}(x, y)$ ) along the cut

$$
\left\{(x, y) \in \mathbb{C}^{2} ; \Im y=\Im s(x), \mathfrak{R} y \geq \mathfrak{R} s(x)\right\}
$$




$$
\left(\text { resp. }\left\{(x, y) \in \mathbf{C}^{2} ; \Im y=\Im(-s(x)), \mathfrak{R} y \geq \mathfrak{R}(-s(x))\right\}\right)
$$

coincides with

$$
\begin{gathered}
2 i \cos \left(\pi \sqrt{\left.1+4 Q_{2}(0)\right)} \psi_{-, B}(x, y)\right. \\
\left(\text { resp. } 2 i \cos \left(\pi \sqrt{\left.1+4 Q_{2}(0)\right)} \psi_{+, B}(x, y)\right) .\right.
\end{gathered}
$$

Remark 2.2. The factor $\sqrt{1+4 Q_{2}(0)}$ in (2.19) and (2.20) is the difference of two characteristic exponents of (2.1) at $x=0$.

In [5] we state that $x=0$ has a similar structure with usual turning points. Theorem 2.1 justifies this statement to the effect that two singular points $y=-s(x)$ and $y=s(x)$ of the Borel transform $\psi_{ \pm, B}(x, y)$ actually merge at $x=0$. Similarly the following definition of the Stokes curve

$$
\Im \int_{0}^{x} \sqrt{\frac{Q_{0}(x)}{x}} d x=0
$$

for (2.1) given in [5] can be justified by the above theorem; Theorem 2.1 tells us that a singular point $y=s(x)$ of $\psi_{+, B}(x, y)$ lies on the path of integration of the Borel sum of $\psi_{+}$if $\mathfrak{R} \int_{0}^{x} \sqrt{Q_{0}(x) / x} d x>0$.

Thus, if we assume the Borel summability of $\psi_{ \pm}$, we obtain from Theorem 2.1 the following connection formula on the Stokes curve (2.21): In case $\mathfrak{R} \int_{0}^{x} \sqrt{Q_{0}(x) / x} d x>0$ along the Stokes curve, we have

$$
\left\{\begin{array}{l}
\psi_{+} \rightarrow \psi_{+}+2 i \cos \left(\pi \sqrt{1+4 Q_{2}(0)}\right) \psi_{-}, \\
\psi_{-} \rightarrow \psi_{-},
\end{array}\right.
$$

i.e., the Borel sum of $\psi_{+}$becomes the Borel sum of $\psi_{+}+$ $2 i \cos \left(\pi \sqrt{1+4 Q_{2}(0)}\right) \psi_{-}$while the Borel sum of $\psi_{-}$is unchanged when we cross the Stokes curve in a counterclockwise manner with respect to the center $x=0$. Similarly, in case $\mathfrak{R} \int_{0}^{x} \sqrt{Q_{0}(x) / x} d x<0$ along the Stokes curve, we have

$$
\left\{\begin{array}{l}
\psi_{+} \rightarrow \psi_{+}, \\
\psi_{-} \rightarrow \psi_{-}+2 i \cos \left(\pi \sqrt{1+4 Q_{2}(0)}\right) \psi_{+}
\end{array}\right.
$$

when we cross the Stokes curve in a counterclockwise manner with respect to the center $x=0$.

\section{§3. Connection Formula for the Borel Transform of WKB Solutions}

The proof of Theorem 2.1 is based on the transformation theory developed in [1]; the idea is to reduce the equation in question to a canonical equation near the origin. 
As discussed in [5] (and also explained in Introduction), the canonical equation of (2.1) near $x=0$ is

$$
\left(-\frac{d^{2}}{d x^{2}}+\eta^{2}\left(\frac{1}{x}+\eta^{-2} \frac{\lambda}{x^{2}}\right)\right) \psi=0,
$$

where $\lambda$ is a constant $Q_{2}(0)$ (cf. Proposition 3.1 below).

For (3.1) we can explicitly describe the Borel transform of WKB solutions $\psi_{ \pm}$in terms of Gauss' hypergeometric functions as follows:

$$
\begin{gathered}
\psi_{+, B}(x, y)=\left.\frac{1}{\sqrt{4 \pi}} s^{-1 / 2} F\left(\alpha-\frac{1}{2}, \beta-\frac{1}{2}, \frac{1}{2} ; s\right)\right|_{s=(y /(4 \sqrt{x}))+(1 / 2)} \\
\psi_{-, B}(x, y)=\left.\frac{1}{\sqrt{-4 \pi}}(1-s)^{-1 / 2} F\left(\alpha-\frac{1}{2}, \beta-\frac{1}{2}, \frac{1}{2} ; 1-s\right)\right|_{s=(y /(4 \sqrt{x}))+(1 / 2)},
\end{gathered}
$$

where $\alpha$ and $\beta$ are constants satisfying

$$
\alpha+\beta=2, \quad \alpha \beta=-4 \lambda .
$$

Furthermore it follows from Gauss' connection formula for hypergeometric functions that

$$
\begin{aligned}
& \psi_{+, B}(x, y)=i \cos (\pi \sqrt{1+4 \lambda}) \psi_{-, B}(x, y)+h_{-}(x, y), \\
& \psi_{-, B}(x, y)=i \cos (\pi \sqrt{1+4 \lambda}) \psi_{+, B}(x, y)+h_{+}(x, y),
\end{aligned}
$$

where $h_{+}(x, y)$ and $h_{-}(x, y)$ are expressed as

$$
h_{+}(x, y)=C_{1} F\left(\alpha, \beta, \frac{3}{2} ; s\right), \quad h_{-}(x, y)=C_{2} F\left(\alpha, \beta, \frac{3}{2} ; 1-s\right) \quad\left(s=\frac{y}{4 \sqrt{x}}+\frac{1}{2}\right)
$$

with some constants $C_{1}$ and $C_{2}$. Hence we can verify Theorem 2.1 for the canonical equation (3.1) by using this explicit description. (See [5], 33 for the details.)

In a general case, we prove Theorem 2.1 by using the reduction of $(2.1)$ to (3.1) which was formally constructed in [5]. In the following we use $\tilde{x}$ as the independent variable of $(2.1)$ and $\tilde{\psi}_{+}$and $\tilde{\psi}_{-}$as WKB solutions of (2.1).

Proposition 3.1. ([1], Proposition 1.1 and Proposition 2.4.) We can find a neighborhood $U$ of $\tilde{x}=0$ and an infinite series

$$
x=x(\tilde{x}, \eta)=x_{0}(\tilde{x})+\eta^{-1} x_{1}(\tilde{x})+\cdots
$$

where $x_{j}(\tilde{x})(j \geq 0)$ is holomorphic in $U$ and satisfies the following: 
(i) $x_{0}(0)=0,\left(d x_{0} / d \tilde{x}\right)(0) \neq 0$.

(ii) $x_{j}(\tilde{x})(j \geq 1)$ vanishes at the origin.

(iii) $x_{2 j-1}(\tilde{x})(j \geq 1)$ is identically zero.

(iv) There exist positive constants $A, C$ for which the following inequality holds in $U$ :

$$
\left|x_{j}(\tilde{x})\right| \leq A C^{j-1} j !
$$

(v) The following relation holds degree by degree with respect to $\eta$ :

$$
\begin{aligned}
& \frac{Q_{0}(\tilde{x})}{\tilde{x}}+\eta^{-2} \frac{Q_{2}(\tilde{x})}{\tilde{x}^{2}} \\
& =\left(\frac{\partial x}{\partial \tilde{x}}\right)^{2}\left(\frac{1}{x(\tilde{x}, \eta)}+\eta^{-2} \frac{\lambda}{x(\tilde{x}, \eta)^{2}}\right)-\frac{1}{2} \eta^{-2}\{x(\tilde{x}, \eta) ; \tilde{x}\}
\end{aligned}
$$

where $\{x ; \tilde{x}\}$ denotes the Schwarzian derivative, i.e.,

$$
\{x ; \tilde{x}\}=\frac{x^{\prime \prime \prime}}{x^{\prime}}-\frac{3}{2}\left(\frac{x^{\prime \prime}}{x^{\prime}}\right)^{2} .
$$

(vi)

$$
\tilde{\psi}_{ \pm}(\tilde{x}, \eta)=\left(\frac{\partial x}{\partial \tilde{x}}(x, \eta)\right)^{-1 / 2} \psi_{ \pm}(x(\tilde{x}, \eta), \eta),
$$

where $\tilde{\psi}_{ \pm}$and $\psi_{ \pm}$are WKB solutions of (2.1) and (3.1) normalized at the origin, respectively.

For reader's convenience we sketch how to construct $x_{j}(\tilde{x})$ (see [5], $\S 2$ for the details).

By substituting (3.8) into (3.10) and comparing both sides degree by degree with respect to $\eta$, we obtain

$$
\frac{1}{x_{0}}\left(\frac{d x_{0}}{d \tilde{x}}\right)^{2}=\frac{Q_{0}(\tilde{x})}{\tilde{x}}
$$

for the 0 -th degree, and

$$
\left(2 \frac{x_{0}^{\prime}}{x_{0}} \frac{d}{d \tilde{x}}-\left(\frac{x_{0}^{\prime}}{x_{0}}\right)^{2}\right) x_{n}(\tilde{x})=F_{n}(\tilde{x})-\delta_{n, 2}\left(\frac{x_{0}^{\prime}}{x_{0}}\right)^{2} \lambda
$$

for the $n$-th degree $(n \geq 1)$. Here $F_{n}(\tilde{x})$ is a rational function of $x_{0}(\tilde{x})$, $x_{1}(\tilde{x}), \ldots, x_{n-1}(\tilde{x})$. Then we find

$$
x_{0}(\tilde{x})=\left(\frac{1}{2} \int_{0}^{\tilde{x}} \sqrt{\frac{Q_{0}(\tilde{x})}{\tilde{x}}} d \tilde{x}\right)^{2}
$$


is a holomorphic solution of (3.13.0) which satisfies the required condition. Note that $2 \sqrt{x_{0}(\tilde{x})}$ coincides with $s(x)$ defined in (2.11).

For $n \geq 1$ we can determine $x_{n}(\tilde{x})$ recursively by solving (3.13.n). Note that in this process we must choose

$$
\lambda=\left.\tilde{x}^{2} F_{2}(\tilde{x})\right|_{\tilde{x}=0} \quad\left(=Q_{2}(0)\right)
$$

to ensure that $x_{2}(\tilde{x})$ vanishes at the origin; otherwise we cannot obtain a holomorphic solution of (3.13.4) (or, at least, (3.13.6)). Due to this choice of $\lambda$, we can recursively determine $x_{n}(\tilde{x})$ which vanishes at the origin and show that $F_{n+1}(\tilde{x})$ has an at most simple pole at the origin.

We now begin the proof of Theorem 2.1, using Proposition 3.1. To give an analytic meaning to (3.12) we first take the Borel transform of both sides. Let $x=x(\tilde{x}, \eta)$ be the infinite series given in Proposition 3.1. Then we can find the following:

$$
\begin{aligned}
\psi_{+}(x(\tilde{x}, \eta), \eta)= & \sum_{n=0}^{\infty}\left[\frac{1}{n !}\left(\sum_{j=1}^{\infty} x_{j}(\tilde{x}) \eta^{-j}\right)^{n}\left(\frac{\partial}{\partial x}\right)^{2} \psi_{+}(x, \eta)\right]_{x=x_{0}(\tilde{x})} \\
= & \sum_{N=0}^{\infty} \eta^{-N} \sum_{\substack{\mu+m=N \\
\mu, m \geq 0}} \sum_{\substack{\mu_{1}+\cdots+\mu_{m}=\mu \\
\mu_{j} \geq 0}} \frac{1}{m !} x_{\mu_{1}+1}(\tilde{x}) \cdots x_{\mu_{m}+1}(\tilde{x}) \\
& \times\left.\left(\frac{\partial}{\partial x}\right)^{m} \psi_{+}(x, \eta)\right|_{\substack{x=x_{0}(\tilde{x}) \\
.}}
\end{aligned}
$$

Hence the right-hand side of (3.12) for $\psi_{+}$becomes

$$
\begin{aligned}
\left(\frac{\partial x}{\partial \tilde{x}}(\tilde{x}, \eta)\right)^{-1 / 2} \psi_{+}(x(\tilde{x}, \eta), \eta) & \frac{1}{\sqrt{x_{0}^{\prime}(\tilde{x})}} \sum_{N=0}^{\infty} \eta^{-N} \sum_{\substack{\mu+v+m+n=N \\
\mu, v \geq 0 \\
m, n \geq 0}} \sum_{\substack{\mu_{1}+\cdots+\mu_{m}=\mu \\
v_{1}+\cdots+v_{n}=v \\
\mu_{j}, v_{j} \geq 0}}(-1)^{n} \frac{\Gamma(n+1 / 2)}{\Gamma(1 / 2) m ! n !} \\
& \times x_{\mu_{1}+1}(\tilde{x}) \cdots x_{\mu_{m}+1}(\tilde{x}) \frac{x_{v_{1}+1}^{\prime}(\tilde{x}) \cdots x_{v_{n}+1}^{\prime}(\tilde{x})}{\left(x_{0}^{\prime}(\tilde{x})\right)^{n}} \\
& \times\left.\left(\frac{\partial}{\partial x}\right)^{m} \psi_{+}(x, \eta)\right|_{x=x_{0}(\tilde{x})} \cdot
\end{aligned}
$$

In order to give a concrete description of the Borel transform of the right-hand side of (3.17), we prepare the following lemma: 
Lemma 3.2. Using the notations introduced in $\S 2$, we find the following relations for an infinite series $\phi(x, \eta)$ of the form (2.9) satisfying (2.12):

(i)

$$
\left[\left(\frac{\partial}{\partial x}\right)^{m} \phi(x, \eta)\right]_{B}=\left(\frac{\partial}{\partial x}\right)^{m} \phi_{B}(x, y) \quad(m=0,1,2, \ldots)
$$

(ii)

$$
\left[\eta^{-n} \phi(x, \eta)\right]_{B}=\frac{1}{(n-1) !} \int_{-s(x)}^{y}(y-t)^{n-1} \phi_{B}(x, t) d t \quad(n=0,1,2, \ldots),
$$

where the integral in the right-hand side of (3.19) should be regarded as a contour integral (around the endpoint $y=-s(x))$.

Remark 3.3. In the following we use the following notation to specify the endpoint of the integral:

$$
\left(\frac{\partial}{\partial y}\right)_{y_{0}}^{-n} \varphi(x, y)=\frac{1}{(n-1) !} \int_{y_{0}}^{y}(y-t)^{n-1} \varphi(x, t) d t
$$

For example, the right-hand side of (3.19) is denoted by $\left(\frac{\partial}{\partial y}\right)_{-s(x)}^{-n} \phi_{B}(x, y)$ in this notation.

Proof of Lemma 3.2. As (i) is obvious, we prove (ii). It follows from the definition of the Borel transform of $\eta^{-n} \phi(x, \eta)$ that

$$
\left[\eta^{-n} \phi(x, \eta)\right]_{B}=\sum_{j=0}^{\infty} \frac{\phi_{j}(x)}{\Gamma(j+n+\alpha)}(y+s(x))^{j+n+\alpha-1} .
$$

Since

$$
\begin{aligned}
& \frac{1}{\Gamma(j+n+\alpha)}(y+s(x))^{j+n+\alpha-1} \\
& \quad=\frac{1}{\Gamma(j+\alpha)} \int_{-s(x)}^{y} \frac{(y-t)^{n-1}}{(n-1) !}(t+s(x))^{j+\alpha-1} d t
\end{aligned}
$$

holds, we obtain

$$
\begin{aligned}
{\left[\eta^{-n} \phi(x, \eta)\right]_{B} } & =\left(\frac{\partial}{\partial y}\right)_{-s(x)}^{-n} \sum_{j=0}^{\infty} \frac{\phi_{j}(x)}{\Gamma(j+\alpha)}(y+s(x))^{j+\alpha-1} \\
& =\left(\frac{\partial}{\partial y}\right)_{-s(x)}^{-n} \phi_{B}(x, y) .
\end{aligned}
$$

Thus we have completed the proof. 
It follows from (3.12), (3.17) and Lemma 3.2 that the Borel transform of $\tilde{\psi}_{+}$ becomes

$$
\begin{aligned}
\tilde{\psi}_{+, B}(\tilde{x}, y)= & \frac{1}{\sqrt{x_{0}^{\prime}(\tilde{x})}} \sum_{N=0}^{\infty} \sum_{\substack{\mu+v+m+n=N \\
\mu, v \geq 0 \\
m, n \geq 0}} \sum_{\substack{\mu_{1}+\cdots+\mu_{m}=\mu \\
v_{1}+\cdots+v_{n}=v \\
\mu_{j}, v_{j} \geq 0}}(-1)^{n} \frac{\Gamma(n+1 / 2)}{\Gamma(1 / 2) m ! n !} \\
& \times x_{\mu_{1}+1}(\tilde{x}) \cdots x_{\mu_{m}+1}(\tilde{x}) \frac{x_{v_{1}+1}^{\prime}(\tilde{x}) \cdots x_{v_{n}+1}^{\prime}(\tilde{x})}{\left(x_{0}^{\prime}(\tilde{x})\right)^{n}} \\
& \times\left.\left(\frac{\partial}{\partial x}\right)^{m}\left(\frac{\partial}{\partial y}\right)_{-2 \sqrt{x}}^{-N} \psi_{+, B}(x, y)\right|_{x=x_{0}(\tilde{x})} .
\end{aligned}
$$

In what follows we take $x=x_{0}(\tilde{x})$ as a new local coordinate and write the above relation $(3.24)$ as

$$
\tilde{\psi}_{+, B}(\tilde{x}, y)=\left.\left(P_{-2 \sqrt{x}} \psi_{+, B}(x, y)\right)\right|_{x=x_{0}(\tilde{x})},
$$

where

$$
\begin{aligned}
& P_{y_{0}}\left(x ; \frac{\partial}{\partial x}, \frac{\partial}{\partial y}\right) \\
& =J(x) \sum_{N=0}^{\infty} \sum_{\substack{\mu+v+m+n=N \\
\mu, v \geq 0 \\
m, n \geq 0}} \sum_{\substack{\mu_{1}+\cdots+\mu_{n}=\mu \\
v_{1}+\cdots+v_{n}=v \\
\mu_{J}, v_{J} \geq 0}}(-1)^{n} \frac{\Gamma(n+1 / 2)}{\Gamma(1 / 2) m ! n !} \\
& \quad \times \tilde{x}_{\mu_{1}+1}(x) \cdots \tilde{x}_{\mu_{m}+1}(x) \tilde{x}_{v_{1}+1}^{\prime}(x) \cdots \tilde{x}_{v_{n}+1}^{\prime}(x)\left(\frac{\partial}{\partial x}\right)^{m}\left(\frac{\partial}{\partial y}\right)_{y_{0}}^{-N}
\end{aligned}
$$

Here we rewrite $x_{j}(\tilde{x})=\tilde{x}_{j}(x)$ and $\left(x_{0}^{\prime}(\tilde{x})\right)^{-1 / 2}=J(x)$ with some holomorphic functions $\tilde{x}_{j}$ and $J$ of $x$.

In a similar way we find

$$
\tilde{\psi}_{-, B}(\tilde{x}, y)=\left.\left(P_{2 \sqrt{x}} \psi_{-, B}(x, y)\right)\right|_{x=x_{0}(\tilde{x})} .
$$

Then Theorem 2.1 (i) immediately follows from the following:

Proposition 3.4. There exists a positive constant $r$ for which $(y+2 \sqrt{x})^{1 / 2} P_{-2 \sqrt{x}} \psi_{+, B}(x, y) \quad$ (resp. $\left.\quad(y-2 \sqrt{x})^{1 / 2} P_{2 \sqrt{x}} \psi_{-, B}(x, y)\right)$ is convergent and defines a holomorphic function in

$$
W_{+}(r)=\left\{(x, y) \in \mathbb{C}^{2} ; 0<|x|<r,|y+2 \sqrt{x}|<4|x|^{1 / 2}\right\}
$$

$$
\text { (resp. } \left.W_{-}(r)=\left\{(x, y) \in \mathbb{C}^{2} ; 0<|x|<r,|y-2 \sqrt{x}|<4|x|^{1 / 2}\right\}\right) \text {. }
$$


Remark 3.5. Since $\psi_{+, B}(x, y)$ has singular points at $y=-2 \sqrt{x}$ and at $y=2 \sqrt{x}$, the above domain of analyticity is the best possible one we can hope.

We shall prove Proposition 3.4 in $\S 4.1$.

Next we consider the analytic continuation of $\tilde{\psi}_{+, B}(\tilde{x}, y)$ by using the relation (3.5) for the Borel transform $\psi_{ \pm, B}(x, y)$ of $\mathrm{WKB}$ solutions of the canonical equation (3.1). In order to consider the action of the operator $P_{y_{0}}$ (defined by (3.26)) to both sides of (3.5), we choose the endpoint $y_{0}$ to be zero; that is, we apply $P_{0}$ to both sides of (3.5) to obtain

$$
P_{0} \psi_{+, B}(x, y)=i \cos \left(\pi \sqrt{1+4 Q_{2}(0)}\right) P_{0} \psi_{-, B}(x, y)+P_{0} h_{-}(x, y) .
$$

Both sides of $(3.30)$ converge in

$$
\left\{(x, y) \in \mathbf{C}^{2} ; 0<|x|<r,|y|<2|x|^{1 / 2}\right\}
$$

if we replace $r$ by a smaller one if necessary. (We can prove this convergence by a similar argument that used in the proof of Proposition 3.4, or by using Proposition 4.1 and Proposition 4.4.)

In order to change the endpoint from $y_{0}=0$ to $y_{0}=-2 \sqrt{x}$ or $y_{0}=2 \sqrt{x}$, let us define

$$
\begin{aligned}
R_{\left[y_{0}, y_{0}^{\prime}\right]}= & J(x) \sum_{N=0}^{\infty} \sum_{\substack{\mu+v+m+n=N \\
\mu, v \geq 0 \\
m, n \geq 0}} \sum_{\substack{\mu_{1}+\cdots+\mu_{n}=\mu \\
v_{1}+\cdots+v_{n}=v \\
\mu_{j}, v_{J} \geq 0}}(-1)^{n} \frac{\Gamma(n+1 / 2)}{\Gamma(1 / 2) m ! n !} \\
& \times \tilde{x}_{\mu_{1}+1}(x) \cdots \tilde{x}_{\mu_{m}+1}(x) \tilde{x}_{v_{1}+1}^{\prime}(x) \cdots \tilde{x}_{v_{n}+1}^{\prime}(x)\left(\frac{\partial}{\partial x}\right)^{m} K_{\left[y_{0}, y_{0}^{\prime}\right]}^{(N)},
\end{aligned}
$$

where

$$
K_{\left[y_{0}, y_{0}^{\prime}\right]}^{(n)} \varphi(x, y)=\frac{1}{(n-1) !} \int_{y_{0}}^{y_{0}^{\prime}}(y-t)^{n-1} \varphi(x, t) d t .
$$

Then we find that $P_{0}=P_{-2 \sqrt{x}}+R_{[0,-2 \sqrt{x}]}$ and $P_{0}=P_{2 \sqrt{x}}+R_{[0,2 \sqrt{x}]}$ hold. Hence from (3.30), we find

$$
P_{-2 \sqrt{x}} \psi_{+, B}(x, y)=i \cos \left(\pi \sqrt{1+4 Q_{2}(0)}\right) P_{2 \sqrt{x}} \psi_{-, B}(x, y)+R_{-}(x, y),
$$

where

$$
\begin{aligned}
R_{-}(x, y)= & -R_{[0,-2 \sqrt{x}]} \psi_{+, B}(x, y)+i \cos \left(\pi \sqrt{1+4 Q_{2}(0)}\right) R_{[0,2 \sqrt{x}]} \psi_{-, B}(x, y) \\
& +P_{2 \sqrt{x}} h_{-}(x, y)+R_{[0,2 \sqrt{x}]} h_{-}(x, y)
\end{aligned}
$$


To examine where $R_{-}(x, y)$ is analytic we prepare the following two propositions:

Proposition 3.6. There exists a positive constant $r$ for which $P_{2 \sqrt{x}} h_{-}(x, y)$ is convergent and defines a holomorphic function in $W_{-}(r)$.

Proposition 3.7. For an arbitrary positive number $L$, there exists a positive constant $r^{\prime}$ for which $R_{[0,-2 \sqrt{x}]} \psi_{+, B}, R_{[0,2 \sqrt{x}]} \psi_{-, B}$ and $R_{[0,2 \sqrt{x}]} h_{-}(x, y)$ are convergent and defines holomorphic functions in

$$
\left\{(x, y) \in \mathbf{C}^{2} ; 0<|x|<r^{\prime},|y+2 \sqrt{x}|<L|x|^{1 / 2}\right\} .
$$

These propositions shall be proved in $\S 4.1$ and $\S 4.2$.

It follows from these two propositions that $R_{-}(x, y)$ is a holomorphic function in $W_{-}(r)$ if we replace $r$ by a smaller one if necessary.

Summing up, we have proved the following. There exists a positive constant $\tilde{r}_{0}$ for which

$$
\tilde{\psi}_{+, B}(\tilde{x}, y)=i \cos \left(\pi \sqrt{1+4 Q_{2}(0)}\right) \tilde{\psi}_{-, B}(\tilde{x}, y)+R_{-}\left(x_{0}(\tilde{x}), y\right)
$$

holds in

$$
\left\{(\tilde{x}, y) \in \mathbf{C}^{2} ; 0<|\tilde{x}|<\tilde{r}_{0},|y|<|s(\tilde{x})|\right\}
$$

and $R_{-}\left(x_{0}(\tilde{x}), y\right)$ is holomorphic in

$$
\tilde{W}_{-}(\tilde{r})=\left\{(\tilde{x}, y) \in \mathbf{C}^{2} ; 0<|\tilde{x}|<\tilde{r}_{0},|y-s(\tilde{x})|<2|s(\tilde{x})|\right\} .
$$

In a similar way we find

$$
\tilde{\psi}_{-, B}(\tilde{x}, y)=i \cos \left(\pi \sqrt{1+4 Q_{2}(0)}\right) \tilde{\psi}_{+, B}(\tilde{x}, y)+R_{+}\left(x_{0}(\tilde{x}), y\right)
$$

holds in

$$
\left\{(\tilde{x}, y) \in \mathbb{C}^{2} ; 0<|\tilde{x}|<\tilde{r}_{0},|y|<|s(\tilde{x})|\right\}
$$

and $R_{+}\left(x_{0}(\tilde{x}), y\right)$ is holomorphic in

$$
\tilde{W}_{+}(\tilde{r})=\left\{(\tilde{x}, y) \in \mathbb{C}^{2} ; 0<|\tilde{x}|<\tilde{r}_{0},|y+s(\tilde{x})|<2|s(\tilde{x})|\right\},
$$

where

$$
\begin{aligned}
R_{+}(x, y)= & -R_{[0,2 \sqrt{x}]} \psi_{-, B}(x, y)+i \cos \left(\pi \sqrt{1+4 Q_{2}(0)}\right) R_{[0,-2 \sqrt{x}]} \psi_{+, B}(x, y) \\
& +P_{-2 \sqrt{x}} h_{+}(x, y)+R_{[0,-2 \sqrt{x}]} h_{+}(x, y) .
\end{aligned}
$$

Theorem 2.1 (ii) and (iii) are immediate consequences of the relations (3.37) and (3.40) and the holomorphy of $R_{ \pm}\left(x_{0}(\tilde{x}), y\right)$. Hence what remains to be proved is Proposition 3.4, Proposition 3.6 and Proposition 3.7. 


\section{§4. Completion of the Proof}

\section{§4.1. Proof of Proposition 3.4 and Proposition 3.6}

For the sake of simplicity we introduce the following notation: For a holomorphic function $f(z)$ on the unit disk $\{z \in \mathbf{C} ;|z|<1\}$ we set

$$
f_{ \pm}(x, y)=f\left( \pm \frac{y}{4 \sqrt{x}}+\frac{1}{2}\right)
$$

and

$$
f_{ \pm}^{(-1 / 2)}(x, y)=\left( \pm \frac{y}{4 \sqrt{x}}+\frac{1}{2}\right)^{-1 / 2} f\left( \pm \frac{y}{4 \sqrt{x}}+\frac{1}{2}\right) .
$$

Then Propositions 3.4 and 3.6 are special cases of the following:

Proposition 4.1. There exists a positive constant $r_{0}$ for which the following hold for any holomorphic function $f(z)$ on the unit disk:

(i) $P_{-2 \sqrt{x}} f_{+}(x, y)$ (resp. $\quad P_{2 \sqrt{x}} f_{-}(x, y)$ ) defines a holomorphic function in $W_{+}\left(r_{0}\right)\left(\right.$ resp. $\left.W_{-}\left(r_{0}\right)\right)$;

(ii) $\left(\frac{y}{4 \sqrt{x}}+\frac{1}{2}\right)^{1 / 2} P_{-2 \sqrt{x}} f_{+}^{(-1 / 2)}(x, y) \quad\left(\right.$ resp. $\left.\quad\left(-\frac{y}{4 \sqrt{x}}+\frac{1}{2}\right)^{1 / 2} P_{2 \sqrt{x}} f_{-}^{(-1 / 2)}(x, y)\right)$ defines a holomorphic function in $W_{+}\left(r_{0}\right)$ (resp. $\left.W_{-}\left(r_{0}\right)\right)$.

Here we give only the proof of this proposition for $f_{+}(x, y)$ and $f_{+}^{(-1 / 2)}(x, y)$. We can prove for $f_{-}(x, y)$ and $f_{-}^{(-1 / 2)}(x, y)$ in a similar way.

Let us begin the argument by showing the following lemma:

Lemma 4.2. For any positive number $\varepsilon$ and any holomorphic function $f(z)$ on the unit disk, there exists a positive constant $M_{\varepsilon}$ for which the following two inequalities hold for

$$
x \neq 0, \quad\left|\frac{y}{4 \sqrt{x}}+\frac{1}{2}\right| \leq 1-\varepsilon
$$

and for $m=0,1, \ldots, l, l=0,1,2, \ldots$ :

(i)

$$
\left|\left(\frac{\partial}{\partial x}\right)^{m}\left(\frac{\partial}{\partial y}\right)_{-2 \sqrt{x}}^{-l} f_{+}(x, y)\right| \leq M_{\varepsilon} \frac{m !}{l !}\left(\frac{2}{|x|}\right)^{m}\left(5 \sqrt{6}|x|^{1 / 2}\right)^{l}
$$

(ii)

$$
\begin{gathered}
\left|\left(\frac{y}{4 \sqrt{x}}+\frac{1}{2}\right)^{1 / 2}\left(\frac{\partial}{\partial x}\right)^{m}\left(\frac{\partial}{\partial y}\right)_{-2 \sqrt{x}}^{-l} f_{+}^{(-1 / 2)}(x, y)\right| \\
\leq M_{\varepsilon} \frac{m !}{\Gamma(l+1 / 2)}\left(\frac{2}{|x|}\right)^{m}\left(5 \sqrt{6}|x|^{1 / 2}\right)^{l}
\end{gathered}
$$


Proof. Since (i) and (ii) are proved in a similar way, we only give the proof of (ii). By using the Taylor expansion $f(z)=\sum_{n \geq 0} c_{n} z^{n}$, we have

$$
\left(\frac{\partial}{\partial y}\right)_{-2 \sqrt{x}}^{-l} f_{+}^{(-1 / 2)}(x, y)=(4 \sqrt{x})^{l} \sum_{n=0}^{\infty} \frac{\Gamma(n+1 / 2)}{\Gamma(n+l+1 / 2)} c_{n}\left(\frac{y}{4 \sqrt{x}}+\frac{1}{2}\right)^{n+l-1 / 2} \text {. }
$$

Let

$$
\begin{aligned}
h(z) & =\sum_{n=0}^{\infty} \frac{\Gamma(n+1 / 2)}{\Gamma(n+l+1 / 2)} c_{n} z^{n+l-1 / 2}, \\
h_{+}^{(-1 / 2)}(x, y) & =h\left(\frac{y}{4 \sqrt{x}}+\frac{1}{2}\right) .
\end{aligned}
$$

Then we have

$$
\begin{aligned}
\left(\frac{\partial}{\partial x}\right)^{m} & \left(\frac{\partial}{\partial y}\right)_{-2 \sqrt{x}}^{-l} f_{+}^{(-1 / 2)}(x, y) \\
= & \sum_{j=0}^{m} \frac{m !}{j !(m-j) !}\left[\left(\frac{\partial}{\partial x}\right)^{j}(4 \sqrt{x})^{l}\right]\left[\left(\frac{\partial}{\partial x}\right)^{m-j} h_{+}^{(-1 / 2)}(x, y)\right] \\
= & \sum_{j=0}^{m} \frac{m !}{j !(m-j) !} \frac{\Gamma(l / 2+1)}{\Gamma(l / 2-j+1)} \frac{(4 \sqrt{x})^{l}}{x^{j}}\left(\frac{\partial}{\partial x}\right)^{m-j} h_{+}^{(-1 / 2)}(x, y) .
\end{aligned}
$$

From the formula of differentiation of composite functions ([7], pp. 6-7), we obtain

$$
\begin{aligned}
& \frac{1}{k !}\left(\frac{\partial}{\partial x}\right)^{k} h_{+}^{(-1 / 2)}(x, y) \\
& \quad=\sum_{j=1}^{k} h^{(j)}\left(\frac{y}{4 \sqrt{x}}+\frac{1}{2}\right) \sum_{\substack{\gamma \in \mathbf{Z}_{+}^{k} \\
\|\gamma\|=k \\
|\gamma|=j}} \prod_{p=1}^{k} \frac{1}{\gamma_{p} !}\left\{\frac{1}{p !}\left(\frac{\partial}{\partial x}\right)^{p}\left(\frac{y}{4 \sqrt{x}}+\frac{1}{2}\right)\right\}^{\gamma_{p}}
\end{aligned}
$$

where $\gamma \in \mathbb{Z}_{+}^{k}$ indicates a multi-index, and

$$
|\gamma|=\sum_{p=1}^{k} \gamma_{p}, \quad\|\gamma\|=\sum_{p=1}^{k} p \cdot \gamma_{p}
$$

To estimate the right-hand side of (4.10) we prepare the following sublemma: 
Sublemma 4.3. The following inequalities hold:

(i)

$$
\sum_{\substack{\gamma \in \mathbf{Z}_{+}^{h} \\\|\gamma\|=k \\|\gamma|=j}} \prod_{p=1}^{k} \frac{1}{\gamma_{p} !}\left|\frac{\Gamma(1 / 2)}{p ! \Gamma(1 / 2-p)}\right|^{\gamma_{p}} \leq \frac{2^{k}}{j !}
$$

(ii)

$$
\sum_{\substack{\gamma \in \mathbf{Z}_{+}^{h} \\ \text { y } y=k \\|\gamma|=j}} \prod_{p=1}^{k} \frac{1}{\gamma_{p} !}\left|\frac{1}{p !}\left(\frac{\partial}{\partial x}\right)^{p}\left(\frac{y}{4 \sqrt{x}}+\frac{1}{2}\right)\right|^{\gamma_{p}} \leq \frac{1}{j !}\left|\frac{y}{4 \sqrt{x}}\right|^{J}\left|\frac{2}{x}\right|^{k} .
$$

Proof of Sublemma 4.3.

(i) Since

$$
\left|\frac{\Gamma(1 / 2)}{p ! \Gamma(1 / 2-p)}\right|=\prod_{J=1}^{p}\left(1-\frac{1}{2 j}\right)<1
$$

we have

$$
\sum_{\substack{\gamma \in \mathbf{Z}_{+}^{h} \\ \text { und } \\|\gamma|=j}} \prod_{p=1}^{k} \frac{1}{\gamma_{p} !}\left|\frac{\Gamma(1 / 2)}{p ! \Gamma(1 / 2-p)}\right|^{\gamma_{p}} \leq \sum_{\substack{\|, \gamma\|=k \\ \gamma, \mid=J}} \frac{1}{\gamma_{1} ! \cdots \gamma_{k} !}
$$

On the other hand

$$
\sum_{\substack{\|\gamma\|=k \\|\gamma|=J}} \frac{1}{\gamma_{1} ! \cdots \gamma_{k} !} \leq \frac{1}{j !} \frac{\left(t+t^{2}+\cdots+t^{k}\right)^{J}}{t^{k}}=\frac{1}{j !} \frac{1}{t^{k}}\left(\frac{t\left(1-t^{k}\right)}{1-t}\right)^{J}
$$

holds for any positive number $t$. Hence we have

$$
\sum_{\substack{\gamma \in \mathbf{Z}_{+}^{k} \\\|\gamma\|=k \\|\gamma|=j}}^{k} \prod_{p=1}^{k} \frac{1}{\gamma_{p} !}\left|\frac{\Gamma(1 / 2)}{p ! \Gamma(1 / 2-p)}\right|^{\gamma_{p}} \leq \frac{1}{j !} \frac{1}{t^{k}}\left(\frac{t\left(1-t^{k}\right)}{1-t}\right)^{J} .
$$

By setting $t=1 / 2$ we obtain (i).

(ii) Since

$$
\left(\frac{\partial}{\partial x}\right)^{p}\left(\frac{y}{4 \sqrt{x}}+\frac{1}{2}\right)=\frac{\Gamma(1 / 2)}{\Gamma(1 / 2-p)} \frac{y}{4} x^{-p-1 / 2}
$$


we obtain

$$
\begin{aligned}
& \sum_{\substack{\gamma \in \mathbf{Z}_{+}^{k} \\
\|\gamma\|=k \\
|\gamma|=J}} \prod_{p=1}^{k} \frac{1}{\gamma_{p} !}\left|\frac{1}{p !}\left(\frac{\partial}{\partial x}\right)^{p}\left(\frac{y}{4 \sqrt{x}}+\frac{1}{2}\right)\right|^{\gamma_{p}} \\
& \quad=\sum_{\substack{\gamma \in \mathbf{Z}_{+}^{k} \\
\|\gamma\|=k \\
|\gamma|=j}} \prod_{p=1}^{k} \frac{1}{\gamma_{p} !}\left|\frac{\Gamma(1 / 2)}{p ! \Gamma(1 / 2-p)} \frac{y}{4 \sqrt{x}} x^{-p}\right|^{\gamma_{p}} \\
& =\left|\frac{y}{4 \sqrt{x}}\right|^{j}\left|\frac{1}{x}\right|^{k} \sum_{\substack{\gamma \in \mathbf{Z}_{+}^{k} \\
\|\gamma\|=k \\
|\gamma| \|=j}}^{k} \prod_{p=1}^{k} \frac{1}{\gamma_{p} !}\left|\frac{\Gamma(1 / 2)}{p ! \Gamma(1 / 2-p)}\right|^{\gamma_{p}} .
\end{aligned}
$$

The right-hand side of (4.19) is dominated by

$$
\frac{1}{j !}\left|\frac{y}{4 \sqrt{x}}\right|^{j}\left|\frac{2}{x}\right|^{k}
$$

in view of (i). This completes the proof of Sublemma 4.3.

Since

$$
h^{(j)}(z)=\sum_{n=0}^{\infty} \frac{\Gamma(n+1 / 2)}{\Gamma(n+l-j+1 / 2)} c_{n} z^{n+l-\jmath-1 / 2},
$$

we have

$$
\left|h^{(j)}(z)\right| \leq \frac{M_{\varepsilon}|z|^{-1 / 2}}{\Gamma(l-j+1 / 2)},
$$

where $M_{\varepsilon}=\sqrt{\pi} \sum_{n \geq 0}\left|c_{n}\right|(1-\varepsilon)^{n}$. It follows from (4.10), (4.22) and Sublemma 4.3 (ii) that the right-hand side of (4.10) is now dominated by

$$
\begin{aligned}
\sum_{j=1}^{k} & \frac{M_{\varepsilon}}{\Gamma(l-j+1 / 2)}\left|\frac{y}{4 \sqrt{x}}+\frac{1}{2}\right|^{-1 / 2} \times \frac{1}{j !}\left|\frac{y}{4 \sqrt{x}}\right|^{j}\left|\frac{2}{x}\right|^{k} \\
& =M_{\varepsilon}\left|\frac{y}{4 \sqrt{x}}+\frac{1}{2}\right|^{-1 / 2}\left|\frac{2}{x}\right|^{k} \sum_{j=1}^{k} \frac{1}{j ! \Gamma(l-j+1 / 2)}\left|\frac{y}{4 \sqrt{x}}\right|^{j} \\
& \leq \frac{M_{\varepsilon}}{\Gamma(l+1 / 2)}\left|\frac{y}{4 \sqrt{x}}+\frac{1}{2}\right|^{-1 / 2}\left|\frac{2}{x}\right|^{k}\left(\frac{5}{2}\right)^{l} .
\end{aligned}
$$

Thus we have finished the estimation of (4.10). 
Applying this estimate to (4.9), we obtain

$$
\begin{aligned}
\mid\left(\frac{y}{4 \sqrt{x}}\right. & \left.+\frac{1}{2}\right)^{1 / 2}\left(\left(\frac{\partial}{\partial x}\right)^{m}\left(\frac{\partial}{\partial y}\right)_{-2 \sqrt{x}}^{l} f_{+}^{(-1 / 2)}\right)(x, y) \mid \\
\leq & \sum_{j=0}^{m} \frac{m !}{j !(m-j) !} \times \frac{\Gamma(l / 2+1)}{\Gamma(l / 2-j+1)} \frac{|4 \sqrt{x}|^{l}}{|x|^{j}} \\
& \times M_{\varepsilon} \frac{(m-j) !}{\Gamma(l+1 / 2)}\left|\frac{2}{x}\right|^{m-j}\left(\frac{5}{2}\right)^{l} \\
\leq & M_{\varepsilon} \frac{m !}{\Gamma(l+1 / 2)}\left|\frac{2}{x}\right|^{m}|10 \sqrt{x}|^{l} \sum_{j=0}^{m} \frac{\Gamma(l / 2+1)}{j ! \Gamma(l / 2-j+1)}\left(\frac{1}{2}\right)^{j} \\
\leq & M_{\varepsilon} \frac{m !}{\Gamma(l+1 / 2)}\left|\frac{2}{x}\right|^{m}\left(5 \sqrt{6}|x|^{1 / 2}\right)^{l} .
\end{aligned}
$$

This finishes the proof of Lemma 4.2(ii).

Now we return to the proof of Proposition 4.1. We first estimate $J(x)=\left(x_{0}^{\prime}(\tilde{x})\right)^{-1 / 2}$ and $\tilde{x}_{j}(x)=x_{j}(\tilde{x})$ constructed in Proposition 3.1. Here we are using a new local coordinate $x=x_{0}(\tilde{x})$. By Proposition 3.1, we can find positive constants $A, C, C_{0}, R$ for which the following hold for $|x|<R$ :

$$
\begin{gathered}
|J(x)|<C_{0}, \\
\left|\tilde{x}_{j}(x)\right| \leq A C^{j-1} j ! \quad(j=0,1,2 \ldots), \\
\left|\tilde{x}_{j}^{\prime}(x)\right| \leq A C^{j-1} j ! \quad(j=0,1,2 \ldots) .
\end{gathered}
$$

Then we have

$$
\begin{aligned}
\mid\left(\frac{y}{4 \sqrt{x}}\right. & \left.+\frac{1}{2}\right)^{1 / 2} P_{-2 \sqrt{x}} f_{+}^{(-1 / 2)}(x, y) \mid \\
\leq & C_{0} \sum_{N=0}^{\infty} \sum_{\substack{a+v+m+n=N \\
\mu, v \geq 0 \\
m, n \geq 0}} \sum_{\substack{\mu_{1}+\cdots+\mu_{m}=\mu \\
v_{1}+\cdots+v_{n}=v \\
\mu_{j}, v_{J} \geq 1}} \frac{\Gamma(n+1 / 2)}{\Gamma(1 / 2) m ! n !} \\
& \times\left(\mu_{1}+1\right) !\left(\mu_{2}+1\right) ! \cdots\left(\mu_{m}+1\right) ! A^{m} C^{\mu} \\
& \times\left(v_{1}+1\right) !\left(v_{2}+1\right) ! \cdots\left(v_{n}+1\right) ! A^{n} C^{v} \\
& \times M_{\varepsilon} \frac{m !}{\Gamma(N+1 / 2)}\left(\frac{2}{|x|}\right)^{m}\left(5 \sqrt{6}|x|^{1 / 2}\right)^{N} .
\end{aligned}
$$


Here we have used (4.25), (4.26), (4.27) and the fact that $\tilde{x}_{1}(x)$ is identically zero. By using the inequality

$$
\sum_{\substack{\mu_{1}+\cdots+\mu_{m}=\mu \\ \mu_{J} \geq 1}}\left(\mu_{1}+1\right) ! \cdots\left(\mu_{m}+1\right) ! \leq 2^{m-1}(\mu+1) !
$$

(which can be readily shown by induction on $m$ ), we obtain

$$
\begin{aligned}
\left|\left(\frac{y}{4 \sqrt{x}}+\frac{1}{2}\right)^{1 / 2} P_{-2 \sqrt{x}} f_{+}^{(-1 / 2)}(x, y)\right| \\
\leq \frac{M_{\varepsilon} C_{0}}{\Gamma(1 / 2)} \sum_{\substack { N=0 \\
\begin{subarray}{c}{\mu+v+m+n=N \\
\mu, v \geq 0 \\
m, n \geq 0{ N = 0 \\
\begin{subarray} { c } { \mu + v + m + n = N \\
\mu , v \geq 0 \\
m , n \geq 0 } }\end{subarray}} \frac{\Gamma(n+1 / 2)}{n ! \Gamma(N+1 / 2)} \times 2^{m-1}(\mu+1) ! C^{\mu} \\
\quad \times 2^{n-1}(v+1) ! A^{n} C^{v}\left(\frac{2 A}{|x|}\right)^{m}\left(5 \sqrt{6}|x|^{1 / 2}\right)^{N} \\
=\frac{M_{\varepsilon} C_{0}}{4 \Gamma(1 / 2)}\left|\frac{y}{4 \sqrt{x}}+\frac{1}{2}\right|^{-1 / 2} \sum_{\mu, v, m, n \geq 0} \frac{\Gamma(n+1 / 2)}{n !} \frac{(\mu+1) !(v+1) !}{\Gamma(\mu+v+m+n+1 / 2)} \\
\quad \times\left(5 \sqrt{6} C|x|^{1 / 2}\right)^{\mu+v}\left(\frac{20 \sqrt{6} A}{|x|^{1 / 2}}\right)^{m}\left(10 \sqrt{6} A|x|^{1 / 2}\right)^{n} .
\end{aligned}
$$

Hence, if we choose $r_{0}=\min \left\{(5 \sqrt{6} C)^{-2}, R\right\},(4.30)$ converges for $0<|x|<r_{0}$. This proves Proposition 4.1 for $f_{+}^{(-1 / 2)}(x, y)$. In a similar way we can prove Proposition 4.1 for $f_{+}(x, y)$ by using Lemma 4.2 (i).

\section{§4.2. Proof of Proposition 3.7}

We use the same notation as in $\S 4.1$ in this subsection. Proposition 3.7 is a special case of the following:

Proposition 4.4. For an arbitrary positive number $L$, there exists a positive constant $r_{0}^{\prime}$ for which $R_{[-2 \sqrt{x}, 0]} f_{+}(x, y)$ and $R_{[-2 \sqrt{x}, 0]} f_{+}^{(-1 / 2)}(x, y)$ (resp. $R_{[2 \sqrt{x}, 0]} f_{-}(x, y)$ and $\left.R_{[2 \sqrt{x}, 0]} f_{-}^{(-1 / 2)}(x, y)\right)$ define holomorphic functions in

$$
\begin{gathered}
\left\{(x, y) \in \mathbb{C}^{2} ; 0<|x|<r_{0}^{\prime},\left|\frac{y}{4 \sqrt{x}}+\frac{1}{2}\right|<L\right\} \\
\left(\operatorname{resp} .\left\{(x, y) \in \mathbb{C}^{2} ; 0<|x|<r_{0}^{\prime},\left|\frac{y}{4 \sqrt{x}}-\frac{1}{2}\right|<L\right\}\right)
\end{gathered}
$$

for any holomorphic function $f(z)$ on the unit disk. 
As in $\S 4.1$, we prove Proposition 4.4 only for $f_{+}(x, y)$ and $f_{+}^{(-1 / 2)}(x, y)$. First we prepare the following lemma:

Lemma 4.5. For any holomorphic function $f(z)$ on the unit disk, there exists a positive constant $M$ for which the following estimates (i) and (ii) hold for $x \neq 0, l=1,2, \ldots$ and $m=0,1, \ldots, l$ :

(i)

$$
\begin{aligned}
& \left|\left(\frac{\partial}{\partial x}\right)^{m} K_{[-2 \sqrt{x}, 0]}^{(l)} f_{+}(x, y)\right| \\
& \quad \leq M \frac{m !}{(l-1) !}\left(\frac{2}{|x|}\right)^{m}\left(4 \sqrt{6}|x|^{1 / 2}\left(\left|\frac{y}{4 \sqrt{x}}+\frac{1}{2}\right|+\frac{1}{2}\right)\right)^{l-1}
\end{aligned}
$$

(ii)

$$
\begin{aligned}
& \left|\left(\frac{\partial}{\partial x}\right)^{m} K_{[-2 \sqrt{x}, 0]}^{(l)} f_{+}^{(-1 / 2)}(x, y)\right| \\
& \quad \leq M \frac{m !}{(l-1) !}\left(\frac{2}{|x|}\right)^{m}\left(4 \sqrt{6}|x|^{1 / 2}\left(\left|\frac{y}{4 \sqrt{x}}+\frac{1}{2}\right|+\frac{1}{2}\right)\right)^{l-1}
\end{aligned}
$$

Proof. We prove the inequality (ii). Let $f(z)=\sum_{n \geq 0} c_{n} z^{n}$. Then

$$
\begin{aligned}
& K_{[-2 \sqrt{x}, 0]}^{(l)} f_{+}^{(-1 / 2)}(x, y) \\
& \quad=\sum_{n=0}^{\infty} \frac{c_{n}}{(l-1) !} \int_{-2 \sqrt{x}}^{0}(y-t)^{l-1}\left(\frac{t}{4 \sqrt{x}}+\frac{1}{2}\right)^{n-1 / 2} d t \\
& =(4 \sqrt{x})^{l} \sum_{j=0}^{l-1} \frac{d_{j}}{j !(l-j-1) !}\left(\frac{y}{4 \sqrt{x}}+\frac{1}{2}\right)^{l-j-1}
\end{aligned}
$$

where

$$
d_{j}=(-1)^{j} \sum_{n=0}^{\infty} \frac{c_{n}}{n+j+1 / 2}\left(\frac{1}{2}\right)^{n+j+1 / 2} .
$$

By letting

$$
\begin{aligned}
h(z) & =\sum_{j=0}^{l-1} \frac{d_{j}}{j !(l-j-1) !} z^{l-j-1}, \\
h_{+}(x, y) & =h\left(\frac{y}{4 \sqrt{x}}+\frac{1}{2}\right),
\end{aligned}
$$

we obtain 


$$
\begin{aligned}
& \left(\frac{\partial}{\partial x}\right)^{m} K_{[-2 \sqrt{x}, 0]}^{(l)} f_{+}^{(-1 / 2)}(x, y) \\
& \quad=\sum_{j=0}^{m} \frac{m !}{j !(m-j) !}\left[\left(\frac{\partial}{\partial x}\right)^{j}(4 \sqrt{x})^{l}\right]\left[\left(\frac{\partial}{\partial x}\right)^{m-j} h_{+}(x, y)\right] \\
& \quad=(4 \sqrt{x})^{l} \sum_{j=0}^{m} \frac{m !}{j !(m-j) !} \frac{\Gamma(l / 2+1)}{\Gamma(l / 2-j+1)} x^{-j}\left(\frac{\partial}{\partial x}\right)^{m-j} h_{+}(x, y) .
\end{aligned}
$$

In parallel with $(4.10)$ we obtain

$$
\begin{aligned}
& \frac{1}{k !}\left(\frac{\partial}{\partial x}\right)^{k} h_{+}(x, y) \\
& =\sum_{j=0}^{k} h^{(j)}\left(\frac{y}{4 \sqrt{x}}+\frac{1}{2}\right) \sum_{\substack{\gamma \in \mathbf{Z}_{+}^{k} \\
\|\gamma\|=k \\
|\gamma|=j}} \prod_{p=1}^{k} \frac{1}{\gamma_{p} !}\left\{\frac{1}{p !}\left(\frac{\partial}{\partial x}\right)^{p}\left(\frac{y}{4 \sqrt{x}}+\frac{1}{2}\right)\right\}^{\gamma_{p}} .
\end{aligned}
$$

Since

$$
h^{(j)}(z)=\sum_{n=0}^{l-j-1} \frac{d_{n}}{n !(l-j-n-1) !} z^{l-j-n-1}
$$

and

$$
\left|d_{n}\right| \leq\left(\frac{1}{2}\right)^{n} M^{\prime}
$$

for some positive constant $M$, we have

$$
\begin{aligned}
\left|h^{(j)}(z)\right| & \leq M^{\prime} \sum_{n=0}^{l-j-1} \frac{1}{n !(l-j-n-1) !}\left(\frac{1}{2}\right)^{n}|z|^{l-j-n-1} \\
& =\frac{M^{\prime}}{(l-j-1) !}\left(|z|+\frac{1}{2}\right)^{l-j-1} .
\end{aligned}
$$

It follows from this inequality and Lemma 4.3(ii) that the right-hand side of (4.40) is dominated by

$$
\begin{aligned}
& \sum_{j=0}^{k} \frac{M^{\prime}}{(l-j-1) !}\left(|z|+\frac{1}{2}\right)^{l-j-1} \times \frac{1}{j !}\left|\frac{y}{4 \sqrt{x}}\right|^{j}\left|\frac{2}{x}\right|^{k} \\
& \quad \leq M^{\prime}\left|\frac{2}{x}\right|^{k} \sum_{j=0}^{k} \frac{1}{j !(l-j-1) !}\left(|z|+\frac{1}{2}\right)^{l-j-1}\left|z-\frac{1}{2}\right|^{j} \\
& \quad \leq \frac{M^{\prime}}{(l-1) !}\left|\frac{2}{x}\right|^{k}(2|z|+1)^{l-1}
\end{aligned}
$$


where $z=\frac{y}{4 \sqrt{x}}+\frac{1}{2}$. Hence we obtain

$$
\begin{aligned}
\left|\left(\frac{\partial}{\partial x}\right)^{m} K_{[-2 \sqrt{x}, 0]}^{(l)} f_{+}^{(-1 / 2)}(x, y)\right| \\
\leq\left(4|x|^{1 / 2}\right)^{l} \sum_{j=0}^{m} \frac{m !}{j !(m-j) !} \frac{\Gamma(l / 2+1)}{\Gamma(l / 2-j+1)}\left|\frac{1}{x}\right|^{j} \\
\quad \times M^{\prime} \frac{(m-j) !}{(l-1) !}\left|\frac{2}{x}\right|^{m-j}(2|z|+1)^{l-1} \\
\leq 4 M^{\prime}|x|^{1 / 2} \frac{m !}{(l-1) !}\left|\frac{2}{x}\right|^{m}\left(8|x|^{1 / 2}\left(|z|+\frac{1}{2}\right)\right)^{l-1} \\
\quad \times \sum_{j=0}^{m} \frac{\Gamma(l / 2+1)}{j ! \Gamma(l / 2-j+1)}\left(\frac{1}{2}\right)^{j} \\
\leq 4 M^{\prime} \sqrt{\frac{2}{3}}|x|^{1 / 2} \frac{m !}{(l-1) !}\left|\frac{2}{x}\right|^{m}\left(4 \sqrt{6}|x|^{1 / 2}\left(|z|+\frac{1}{2}\right)\right)^{l-1} .
\end{aligned}
$$

This proves (ii). In a similar way we can prove (i). Thus the proof has been completed.

Now we prove Proposition 4.4. Let $A, C, C_{0}$ and $R$ be constants given in (4.25), (4.26) and (4.27). Then we have

$$
\begin{aligned}
& \left|R_{[-2 \sqrt{x}, 0]} f_{+}^{(-1 / 2)}(x, y)\right| \\
& \leq C_{0}\left\{1+\sum_{N=1}^{\infty} \sum_{\substack{\mu+v+m+n=N \\
\mu, v \geq 0 \\
m, n \geq 0}} \sum_{\substack{\mu_{1}+\cdots+\mu_{m}=\mu \\
v_{1}+\cdots+v_{n}=v \\
\mu_{j}, v_{j} \geq 1}} \frac{\Gamma(n+1 / 2)}{\Gamma(1 / 2) m ! n !}\right. \\
& \times\left(\mu_{1}+1\right) !\left(\mu_{2}+1\right) ! \cdots\left(\mu_{m}+1\right) ! A^{m} C^{\mu} \\
& \times\left(v_{1}+1\right) !\left(v_{2}+1\right) ! \cdots\left(v_{n}+1\right) ! A^{n} C^{v} \\
& \left.\times M \frac{m !}{(N-1) !}\left(\frac{2}{|x|}\right)^{m}\left(4 \sqrt{6}|x|^{1 / 2}\left(\left|\frac{y}{4 \sqrt{x}}+\frac{1}{2}\right|+\frac{1}{2}\right)\right)^{N-1}\right\}
\end{aligned}
$$




$$
\begin{aligned}
\leq C_{0} & \left\{1+\frac{M}{\Gamma(1 / 2)}\left[4 \sqrt{6}|x|^{1 / 2}\left(\left|\frac{y}{4 \sqrt{x}}+\frac{1}{2}\right|+\frac{1}{2}\right)\right]^{-1}\right. \\
& \times \sum_{\substack { N=1 \\
\begin{subarray}{c}{\mu+v+m+n=N \\
\mu, v \geq 0 \\
m, n \geq 0{ N = 1 \\
\begin{subarray} { c } { \mu + v + m + n = N \\
\mu , v \geq 0 \\
m , n \geq 0 } }\end{subarray}}^{\infty} \frac{\Gamma(n+1 / 2)}{n !(N-1) !} 2^{m-1}(\mu+1) ! C^{\mu} \times 2^{n-1}(v+1) ! A^{n} C^{v} \\
& \left.\times\left(\frac{2 A}{|x|}\right)^{m}\left(4 \sqrt{6}|x|^{1 / 2}\left(\left|\frac{y}{4 \sqrt{x}}+\frac{1}{2}\right|+\frac{1}{2}\right)\right)^{N}\right\} \\
\left.\leq C_{0}\right\} & \left\{\frac{M}{\Gamma(1 / 2)}\left[4 \sqrt{6}|x|^{1 / 2}\left(\left|\frac{y}{4 \sqrt{x}}+\frac{1}{2}\right|+\frac{1}{2}\right)\right]^{-1}\right. \\
& \times \sum_{\substack{\mu, v, m, n \geq 0 \\
\mu+v+m+n \neq 0}} \frac{\Gamma(n+1 / 2)}{n !} \frac{(\mu+1) !(v+1) !}{(\mu+v+m+n-1) !} \\
& \times\left(4 \sqrt{6}|x|^{1 / 2}\left(\left|\frac{y}{4 \sqrt{x}}+\frac{1}{2}\right|+\frac{1}{2}\right)\right)^{\mu+v} \times\left(\frac{16 \sqrt{6} A}{|x|^{1 / 2}}\left(\left|\frac{y}{4 \sqrt{x}}+\frac{1}{2}\right|\right)\right)^{m} \\
& \left.\times\left(8 \sqrt{6} A|x|^{1 / 2}\left(\left|\frac{y}{4 \sqrt{x}}+\frac{1}{2}\right|\right)\right)^{n}\right\} .
\end{aligned}
$$

Hence, if we take $r_{0}^{\prime}=\min \left\{(4 \sqrt{6}(L+1 / 2))^{-2}, R\right\}$ for a given $L$, (4.46) converges for

$$
0<|x|<r_{0}^{\prime}\left|\frac{y}{4 \sqrt{x}}+\frac{1}{2}\right|<L
$$

Thus the proof of Proposition 4.4 has been completed.

\section{References}

[1] Aoki, T., Kawai, T. and Takei, Y., The Bender-Wu analysis and the Voros theory, ICM-90 Satellite Conference Proceedings "Special Functions", Springer-Verlag (1991), 1-29.

[2] —_ Algebraic analysis of singular perturbations, Sügaku Expositions, 8 (1995), 217-240. (Originally appeared in Japanese in Sūgaku, 45 (1993), 299-315.)

[3] Delabaere, D., Dillinger, H. et Pham. F., Résurgence de Voros et périodes des courbes hyperelliptiques, Ann. Inst. Fourier, 43 (1993), 163-199. 
[4] Fedoryuk, M. V., Asymptotic Analysis-Linear Ordinary Differential Equations, SpringerVerlag, 1993.

[5] Koike, T., On a regular singular point in the exact WKB analysis, Toward the Exact WKB Analysis of Differential Equations, Linear or Non-linear. Kyoto Univ. Press, 2000, 39-54.

[6] —- On the asymptotics of the spectrum of Heun's equation and exact WKB analysis, Toward the Exact WKB Analysis of Differential Equations, Linear or Non-linear, Kyoto Univ. Press, 2000, 55-70.

[ 7 ] Moriguchi, S., Utagawa, K. and Hitotsumatsu, S., Mathematical formulas, Vol. I, Iwanami, 1956. (In Japanese.)

[ 8 ] Olver, F. W. J., Asymptotics and Special Functions, A K Peters, Ltd., 1997. Originally published by Academic Press, 1974.

[9] Voros, A., The return of the quartic oscillator, The complex WKB method, Ann. Inst. Henri Poincaré, 39 (1983), 211-338. 
